\title{
ON THE DOMAIN OF SELFADJOINT EXTENSION OF THE PRODUCT OF STURM-LIOUVILLE DIFFERENTIAL OPERATORS
}

\author{
SOBHY EL-SAYED IBRAHIM
}

Received 10 August 2001

\begin{abstract}
The second-order symmetric Sturm-Liouville differential expressions $\tau_{1}, \tau_{2}, \ldots, \tau_{n}$ with real coefficients are considered on the interval $I=(a, b),-\infty \leq a<b \leq \infty$. It is shown that the characterization of singular selfadjoint boundary conditions involves the sesquilinear form associated with the product of Sturm-Liouville differential expressions and elements of the maximal domain of the product operators, and it is an exact parallel of the regular case. This characterization is an extension of those obtained by Everitt and Zettl (1977), Hinton, Krall, and Shaw (1987), Ibrahim (1999), Krall and Zettl (1988), Lee (1975/1976), and Naimark (1968).
\end{abstract}

2000 Mathematics Subject Classification: 34A05, 34B24, 47A55, 47E05.

1. Introduction. In [10], Krall and Zettl considered the Sturm-Liouville differential expression

$$
\tau[y]=\left[-\left(p y^{\prime}\right)^{\prime}+q y\right] \quad \text { on } I=(a, b),-\infty \leq a<b \leq \infty,
$$

with real-valued Lebesgue measurable functions $p$ and $q$ assumed to satisfy the following basic conditions:

$$
p^{-1}, q \in L_{\mathrm{loc}}(I)
$$

and proved that the characterization of the singular selfadjoint boundary conditions is identical to that in the regular case provided that $y$ and $p y^{\prime}$ are replaced by certain Wronskians involving $y$ and two linearly independent solutions of $\tau[y]=0$.

The relationship between the deficiency index of a symmetric differential expression (1.1) and its powers $\tau^{2}, \tau^{3}, \ldots$ has recently been studied by Chaudhuri and Everitt [1], and the relationship between the number of linearly independent $L^{2}(0, \infty)$ solutions of the equations $\tau_{j}[y]=0$ and of the product equations $\left(\tau_{1} \tau_{2} \cdots \tau_{n}\right) y=0$ has been investigated by Everitt and Zettl [4]. These results are an extension of those recently obtained in $[3,15,16,18]$ for the special case $\tau_{j}=\tau$ for $j=1, \ldots, n$, and $\tau$ is a real second-order symmetric differential expression. 
Our objective in this paper is to show that the characterization of the singular selfadjoint boundary conditions is identical to that in the regular case provided that $y$ and its quasiderivatives are replaced by sesquilinear forms associated with the product of Sturm-Liouville differential expressions, involving $y$ and elements of the maximal domain of the product operators. This characterization is an extension of those by Everitt and Zettl [4] and those in $[5,6,7,10,11,12,13]$.

In the regular case, these conditions can be interpreted as linear combinations of the values of the unknown function $y$ and its quasiderivatives at the endpoints $a$ and $b$.

In the singular case, these conditions are given in terms of sesquilinear forms involving $y$ and linearly independent solutions of the product equation $\left(\tau_{1} \tau_{2} \cdots \tau_{n}\right) y=0$ given by Everitt and Zettl in [4].

2. Preliminaries. We begin with a brief summary of adjoint pairs of operators and products operators (a full treatment may be found in [2, Chapter III] and $[3,4,5,7,8,9])$.

The domain and range of a linear operator $T$ acting in a Hilbert space $H$ will be denoted by $D(T)$ and $R(T)$, respectively, and $N(T)$ will denote its null space. The nullity of $T$, written $\operatorname{nul}(T)$, is the dimension of $N(T)$, and the deficiency of $T$, written $\operatorname{def}(T)$, is the codimension of $R(T)$ in $H$; thus, if $T$ is densely defined and $R(T)$ is closed, then $\operatorname{def}(T)=\operatorname{nul}\left(T^{*}\right)$. The Fredholm domain of $T$ is (in the notation of [2]) the open subset $\triangle_{3}(T)$ of $\mathbb{C}$ consisting of those values $\lambda \in \mathbb{C}$ which are such that $T-\lambda I$ is a Fredholm operator. Thus, $\lambda \in \triangle_{3}(T)$ if and only if $(T-\lambda I)$ has a closed range and finite nullity and deficiency, $I$ being the identity operator on $H$. The index of $(T-\lambda I)$ is the number ind $(T-\lambda I)=$ $\operatorname{nul}(T-\lambda I)-\operatorname{def}(T-\lambda I)$, this being defined for $\lambda \in \triangle_{3}(T)$.

A closed operator $A$ in a Hilbert space $H$ has property $(C)$ if it has a closed range and $\lambda=0$ is not an eigenvalue; that is, there is some positive number $r$ such that $\|A x\| \geq r\|x\|$ for all $x \in D(A)$.

Note that property $(C)$ is equivalent to $\lambda=0$, being a regular type point of $A$. This, in turn, is equivalent to the existence of $A^{-1}$ as a bounded operator on the range of $A$ (which need not be all of $H$ ).

Given two operators $A$ and $B$, both acting in a Hilbert space $H$, we wish to consider the product operator $A B$. This is defined as follows:

$$
D(A B)=\{x \in D(A) \mid B x \in D(A)\}, \quad(A B) x=A(B x), \quad \forall x \in D(A B) .
$$

It may happen in general that $D(A B)$ contains only the null element of $H$. However, in the case of many differential operators, the domains of the product will be dense in $H$.

The next result gives conditions under which the deficiency of a product is the sum of the deficiencies of the factors. 
LEMMA 2.1 (cf. [4, Theorem A] and [16]). Let $A$ and $B$ be closed operators with dense domains in a Hilbert space $H$. Suppose that $\lambda=0$ is a regular type point for both operators and $\operatorname{def} A$ and $\operatorname{def} B$ are finite. Then, $A B$ is a closed operator with dense domain and has $\lambda=0$ as a regular type point, and

$$
\operatorname{def} A B=\operatorname{def} A+\operatorname{def} B .
$$

Evidently, Lemma 2.1 extends to the product of any finite number of operators $A_{1}, A_{2}, \ldots, A_{n}$.

Let the interval $I$ have endpoints $a$ and $b(-\infty \leq a<b \leq \infty)$, and let $w: I \rightarrow \mathbb{R}$ be a nonnegative weight function with $w \in L_{\text {loc }}^{1}(I)$ and $w(x)>0$ (for almost all $x \in I$ ). Then, $H=L_{w}^{2}(I)$ denotes the Hilbert function space of equivalence classes of Lebesgue measurable functions such that $\int_{I} w|f|^{2}<\infty$; the innerproduct is defined by

$$
(f, g):=\int_{I} w(x) f(x) \overline{g(x)} d x \quad\left(f, g \in L_{w}^{2}(I)\right) .
$$

We will consider the Sturm-Liouville differential equation of the form

$$
\tau[y]=-\left(p y^{\prime}\right)^{\prime}+q y=\lambda w y \quad \text { on } I
$$

where the real-valued Lebesgue measurable functions $p, q$, and $w$ from $I$ into $\mathbb{R}$ are satisfying conditions (1.2), which are taken to hold throughout this paper. Under these assumptions, $\tau$ is interpreted as a quasidifferential expression, $u$ is a solution of (2.4) if $u$ and $p u^{\prime}$ are in $\operatorname{AC}_{\text {loc }}(a, b)$, the space of functions which are absolutely continuous on compact subsets of $(a, b)$, and (2.4) is satisfied almost everywhere on $(a, b)$. Also, $p u^{\prime}=u^{[1]}$ is called the quasi-derivative of $u$.

Equation (2.4) is said to be regular at the left endpoint $a \in \mathbb{R}$ if, for all $X \in(a, b)$,

$$
a \in \mathbb{R} ; \quad p^{-1}, q, w \in L^{1}[a, X]
$$

otherwise, (2.4) is said to be singular at $a$. If (2.4) is regular at both endpoints $a$ and $b$, then it is said to be regular; in this case we have

$$
a, b \in \mathbb{R} ; \quad p^{-1}, q, w \in L^{1}(a, b) .
$$

We will be concerned with the second-order symmetric differential expression on $I$ and when both endpoints $a$ and $b$ may be either regular or singular endpoints of (2.4). Note that, in view of (1.2), an endpoint of $I$ is regular for (2.4) if and only if it is regular for the equation

$$
\tau^{+}[z]=\bar{\lambda} w z \quad(\lambda \in \mathbb{C}) \text { on } I,
$$


where $\tau^{+}$is the formal, or Lagrangian, adjoint of $\tau$ given by

$$
\tau^{+}[z]=-\left(p z^{\prime}\right)^{\prime}+q z \text { on } I \text {. }
$$

The maximal domain $D(\tau)$, defined by

$$
D(\tau):=\left\{f: f, p f^{\prime} \in \operatorname{AC}_{\mathrm{loc}}(I), w^{-1} \tau[f] \in L_{w}^{2}(a, b)\right\},
$$

is a subspace of $L_{w}^{2}(a, b)$. The maximal operator $T(\tau)$ is defined by

$$
T(\tau) y:=w^{-1} \tau[y] \quad(y \in D(\tau)) .
$$

It is well known that $D(\tau)$ is dense in $L_{w}^{2}(a, b)$, see $[7,8,9,10]$.

In the regular problem, the minimal operator $T_{0}(\tau)$ is the restriction of $w^{-1} \tau[u]$ to the subspace

$$
D_{0}(\tau):=\left\{y: y \in D(\tau), y^{[r-1]}(a)=y^{[r-1]}(b)=0, r=1,2\right\}
$$

The subspace $D_{0}(\tau)$ is dense and closed in $L_{w}^{2}(a, b)$, see $[2,13,17]$.

In the singular problem, we first introduce the operator $T_{0}^{\prime}(\tau), T_{0}^{\prime}(\tau)$ being the restriction of $w^{-1} \tau[\cdot]$ to the subspace

$$
D_{0}^{\prime}(\tau):=\{y: y \in D(\tau), \operatorname{supp} y \subset(a, b)\}
$$

This operator is densely defined and closable in $L_{w}^{2}(a, b)$, and we defined the minimal operator $T_{0}(\tau)$ to be its closure (see [2, 13] and [17, Section 5]). We denote the domain of $T_{0}(\tau)$ by $D_{0}(\tau)$. It can be shown that

$$
y \in D_{0}(\tau) \Longrightarrow y^{[r-1]}(a)=0, \quad(r=1,2),
$$

whenever we assume $a$ to be a regular endpoint and $b$ to be a singular endpoint.

For $f, g \in D(\tau)$ and $\alpha, \beta \in I$, Green's formula is given by

$$
\int_{\alpha}^{\beta}\{\tau[f] \bar{g}-f \overline{\tau[g]}\} d x=[f, g](\beta)-[f, g](\alpha),
$$

where

$$
[f, g]:=f \bar{g}^{[1]}-f^{[1]} \bar{g}, \quad f, g \in D(\tau) .
$$

For $f, g \in D(\tau)$, the limits $\lim _{\alpha \rightarrow a^{+}}[f, g](\alpha)$ and $\lim _{\beta \rightarrow b^{-}}[f, g](\beta)$ exist and are finite. These are denoted by $[f, g](a)$ and $[f, g](b)$, respectively.

For $f, g \in \mathrm{AC}_{\text {loc }}(a, b)$, let

$$
W(f, g)=f p g^{\prime}-g p f^{\prime}
$$


Choose two solutions $\theta$ and $\phi$ of $\tau[u]=0$ satisfying

$$
W(\theta, \phi)(x)=1 \quad \forall x \in I .
$$

Clearly such $\theta$ and $\phi$ exist, that is, they can be determined by the initial conditions $\theta(c)=1,\left(p \theta^{\prime}\right)(c)=1, \phi(c)=0,\left(p \phi^{\prime}\right)(c)=1$ for all $c$ in $I$.

Note that the sesquilinear form $[f, g]$ in $(2.15)$ can be written as

$$
[f, g]=f p \bar{g}^{\prime}-\bar{g} p f^{\prime}=\left(\bar{g}, p \bar{g}^{\prime}\right)\left(\begin{array}{cc}
0 & -1 \\
1 & 0
\end{array}\right)\left(\begin{array}{c}
f \\
p f^{\prime}
\end{array}\right) .
$$

From (2.16) and (2.17), we get

$$
\left(\begin{array}{cc}
0 & -1 \\
1 & 0
\end{array}\right)=-\left(\begin{array}{cc}
0 & -1 \\
1 & 0
\end{array}\right)\left(\begin{array}{cc}
\theta & \phi \\
p \theta^{\prime} & p \phi^{\prime}
\end{array}\right)\left(\begin{array}{cc}
0 & -1 \\
1 & 0
\end{array}\right)\left(\begin{array}{cc}
\theta & p \theta^{\prime} \\
\phi & p \phi^{\prime}
\end{array}\right)\left(\begin{array}{cc}
0 & -1 \\
1 & 0
\end{array}\right)
$$

and hence the sesquilinear form in (2.18) can also be written as

$$
\begin{aligned}
{[f, g] } & =(W(\bar{g}, \theta), W(\bar{g}, \phi))\left(\begin{array}{cc}
0 & -1 \\
1 & 0
\end{array}\right)\left(\begin{array}{l}
W(f, \theta) \\
W(f, \phi)
\end{array}\right) \\
& =W(\bar{g}, \phi) W(f, \theta)-W(\bar{g}, \theta) W(f, \phi) \\
& =\operatorname{det}\left(\begin{array}{ll}
W(f, \theta) & W(f, \phi) \\
W(\bar{g}, \theta) & W(\bar{g}, \phi)
\end{array}\right),
\end{aligned}
$$

see $[7,10]$.

LEMMA 2.2. If, for sum $\lambda_{0} \in \mathbb{C}$, there are two linearly independent solutions of $\tau[y]=\lambda_{0} w y$ in $L_{w}^{2}(a, b)$, then all solutions of $\tau[y]=\lambda w y$ are in $L_{w}^{2}(a, b)$ for all $\lambda \in \mathbb{C}$, see [2, Chapter 3] for more details.

THEOREM 2.3 (cf. [2, Theorem 3.10.1]). Let $f \in L_{\mathrm{loc}}^{1}(a, b)$, and suppose that conditions (1.2) are satisfied. Then, given any complex numbers $c_{0}$ and $c_{1}$ and any $x_{0} \in(a, b)$, there exists a unique solution of $\tau[\phi]=f$ in $(a, b)$ which satisfies $\phi\left(x_{0}\right)=c_{0}$ and $\phi^{[1]}\left(x_{0}\right)=c_{1}$.

A simple consequence of Theorem 2.3 is that the solutions of (2.4) form a two-dimensional vector space over $\mathbb{C}$. If $\left(\alpha_{0}, \alpha_{1}\right)$ and $\left(\beta_{0}, \beta_{1}\right)$ are linearly independent vectors in $C^{2}$, then the solutions $\phi_{1}(\cdot, \lambda)$ and $\phi_{2}(\cdot, \lambda)$ of (2.4), which satisfy $\phi_{1}\left(x_{0}, \lambda\right)=\alpha_{0}, \phi_{1}^{[1]}\left(x_{0}, \lambda\right)=\alpha_{1}, \phi_{2}\left(x_{0}, \lambda\right)=\beta_{0}$ and $\phi_{2}^{[1]}\left(x_{0}, \lambda\right)=$ $\beta_{1}$ for some $x_{0} \in(a, b)$, form a basis for the space of the solutions of (2.4).

Note that an important distinction between a regular endpoint and a singular endpoint is the fact that, at a regular endpoint $x_{0}$, all initial-value problems $\phi\left(x_{0}, \lambda\right)=c_{0}, \phi^{[1]}\left(x_{0}, \lambda\right)=c_{1}$ and $c_{0}, c_{1} \in \mathbb{C}$ have unique solutions. This is not true when $x_{0}$ is a singular endpoint (see $[2,9]$ ).

In the case that $a$ and $b$ are singular endpoints, and for any $\alpha$ and $\beta$ in the open interval $(a, b)$ and any $\lambda \in \mathbb{C}$, conditions (1.2) imply that any solution $\phi$ 
of (2.4) is in $L_{w}^{2}(a, b)$, (see [9, 10, 14]). However, it is possible that such a $\phi$ does not belong to $L_{w}^{2}(a, b)$. If $\phi$ is in $L_{w}^{2}(a, b)$, for some $\beta \in(a, b)$, then this is true for all $\beta$ in $(a, b)$. If all solutions of (2.4) are in $L_{w}^{2}(a, \beta)$, for some $\beta$ in $(a, b)$, then we say that $\tau[\cdot]$ is in the limit-circle case at $a$, or, simply, that $a$ is LC. Otherwise, $\tau[\cdot]$ is in the limit-point case at $a$ or $a$ is LP. Similarly, $b$ is LC means that all solutions of (2.4) are in $L_{w}^{2}(\alpha, b), a<\alpha<b$. This classification is independent of $\lambda$ in (2.4), (see [7, 10, 13, 18]). Otherwise, $b$ is LP. The limitpoint, limit-circle terms are used for historical reasons.

The classification of the selfadjoint extensions of $T_{0}(\tau)$ depends, in an essential way, on the deficiency index of $T_{0}(\tau)$. We briefly recall the definition of this notion for abstract symmetric operators in a separable Hilbert space.

A linear operator $A$ from a Hilbert space $H$ into $H$ is said to be symmetric if its domain $D(A)$ is dense in $H$ and $(A f, g)=(f, A g)$ for all $f, g \in D(A)$. Any such operator has associated with it a pair $\left(d^{+}, d^{-}\right)$, where each of $d^{+}, d^{-}$is a nonnegative or $+\infty$. The extended integers are called the deficiency indices of $A$, and we have the following.

For $\lambda \in \mathbb{C}$, the set of complex numbers, let $R_{\lambda}$ denote the range of $T_{0}(\tau)-\bar{\lambda} I$, $N_{\lambda}=R_{\lambda}^{\perp}$ and let

$$
N^{+}=N_{i}, \quad N^{-}=N_{-i}, \quad i=\sqrt{-1},
$$

$d^{+}=$dimension of $N^{+}$and $d^{-}=$dimension of $N^{-}$. The spaces $N^{+}$and $N^{-}$are called the deficiency spaces of $T_{0}(\tau)$, and $d^{+}$and $d^{-}$are called the deficiency indices of $T_{0}(\tau)$. These are related to (2.4) as follows:

$$
N_{\lambda}=\left\{f \in D\left[T_{0}^{*}(\tau)\right] \mid\left[T_{0}^{*}(\tau)\right] f=[T(\tau)] f=w^{-1} \tau[f]=\lambda f\right\} .
$$

Thus, $N^{+}$and $N^{-}$consist of the solutions of (2.4) which lie in the space $H=$ $L_{w}^{2}(I)$ for $\lambda=+i$ and $\lambda=-i$, respectively. Hence, $d^{+}$and $d^{-}$are the number of linearly independent solutions of (2.4) which are in the space $H$ for $\lambda=+i$ and $\lambda=-i$, respectively. It is clear for a symmetric differential operator $T_{0}(\tau)$ that

$$
0 \leq d^{+}=d^{-} \leq 2
$$

We denote the common value by $d$ and call $d$ the deficiency index of $\tau$ on $I$. From the above discussion, we see that there are only three possibilities for $d$ : $d=0,1,2$.

Note that, in the literature, the maximal and minimal deficiency cases are often referred to as the limit-circle and limit-point cases. Strictly, these latter terms are only suitable for the now classical second-order differential expressions; in this case the terminology was originally introduced by Hermann Weyl. The term limit-point does give an acceptable description of the minimal deficiency case for real, and hence even-order, symmetric expressions.

Now, we recall the following results. 
For any $\lambda \in \mathbb{C} \backslash \mathbb{R}$ and for a symmetric differential operator $T_{0}(\tau)$, we, from the general theory, have

$$
D(\tau)=D_{0}(\tau)+N^{+}+N^{-},
$$

where $D_{0}(\tau), N^{+}$, and $N^{-}$are linearly independent subspaces and the sum is direct (which we indicate with the symbol + ), see [2, 5, 7, 13].

Any selfadjoint extension $S$ of the symmetric differential operator $T_{0}(\tau)$ satisfies

$$
T_{0}(\tau) \subset S=S^{*} \subset T_{0}^{*}(\tau)
$$

and hence is completely determined by specifying its domain $D(S)$,

$$
D\left[T_{0}(\tau)\right] \subset D(S) \subset D\left[T_{0}^{*}(\tau)\right]
$$

This can be proved using formula (2.23) (see [1, 2, 5, 7, 13]).

THEOREM 2.4. The operator $T_{0}(\tau)$ is a closed symmetric operator from $H$ into $H$ and

$$
T_{0}^{*}(\tau)=T(\tau), \quad T^{*}(\tau)=T_{0}(\tau), \quad D_{0}(\tau)=\text { domain of } T^{*}(\tau) .
$$

Proof. See [7, 10] and [13, Section 17.4].

Some of the basic facts are summarized in the following theorem.

THEOREM 2.5 (cf. [10, Proposition 1]). (a) $D_{0}(\tau)=\{f \in D(\tau):[f, g](b)-$ $[f, g](a)=0$ for all $g \in D(\tau)\}$.

(b) If $\tau[\cdot]$ is in the limit-point case at an endpoint $c$, then $[f, g](c)=0$ for all $f, g \in D[T(\tau)], c=a$ or $c=b$.

(c) If an endpoint $c$ is regular, then, for any solution $u, u$ and $u^{[1]}$ are continuous at $c$.

(d) If $a$ and $b$ are both regular endpoints, then, for any $\alpha, \beta, \gamma$, and $\delta$ in $\mathbb{C}$, there exists a function $f$ in $D(\tau)$ such that

$$
\begin{array}{ll}
f(a)=\alpha, & f^{[1]}(a)=\beta, \\
f(b)=\gamma, & f^{[1]}(b)=\delta .
\end{array}
$$

(e) If $a$ is regular and $b$ is singular, then a function $f$ from $D[T(\tau)]$ is in $D\left[T_{0}(\tau)\right]$ if and only if the following conditions are satisfied:

(i) $f(a)=0$ and $f^{[1]}(a)=0$,

(ii) $[f, g](b)=0$ for all $f, g \in D T(\tau)$.

The analogous results hold when $a$ is singular and $b$ is regular, see also [6, 9, 10]. 
LEMmA 2.6 (cf. [7] and [10, Lemma 2]). Given $\alpha, \beta, \gamma$, and $\delta$ in $\mathbb{C}$, then there exists a $\Psi \in D[T(\tau)] \backslash D\left[T_{0}(\tau)\right]$ such that

$$
\begin{array}{ll}
W(\Psi, \theta)(a)=\alpha, & W(\Psi, \phi)(a)=\beta, \\
W(\Psi, \theta)(b)=\gamma, & W(\Psi, \phi)(b)=\delta .
\end{array}
$$

Furthermore, $\Psi$ can be taken to be a linear combination of $\theta$ and $\phi$ near each endpoint.

3. Some technical lemmas. The proof of general theorem will be based on the results in this section. We start by listing some properties and results of Sturm-Liouville differential expressions $\tau_{1}, \tau_{2}, \ldots, \tau_{n}$, each of order two. For proofs, the reader is referred to $[4,7,8,9,15,16,18]$.

$$
\begin{gathered}
\left(\tau_{1}+\tau_{2}\right)^{+}=\tau_{1}^{+}+\tau_{2}^{+}, \\
\left(\tau_{1} \tau_{2}\right)^{+}=\tau_{2}^{+} \tau_{1}^{+}, \quad(\lambda \tau)^{+}=\bar{\lambda} \tau^{+} \text {for } \lambda \text { a complex number. }
\end{gathered}
$$

A consequence of properties (3.1) is that if $\tau^{+}=\tau$ then $P(\tau)^{+}=P\left(\tau^{+}\right)$for $P$ any polynomial with complex coefficients. Also, we note that the leading coefficients of a product is the product of the leading coefficients. Hence, the product of regular differential expressions is regular.

LEMmA 3.1 (cf. [4, Theorem 1]). Suppose that $\tau_{j}$ is a regular differential expression on the interval $[a, b]$ such that the minimal operator $T_{0}\left(\tau_{j}\right)$ has property $(C)$ for $j=1,2, \ldots, n$. Then,

(i) the product operator $\prod_{j=1}^{n}\left[T_{0}\left(\tau_{j}\right)\right]$ is closed and have dense domain, property $(C)$, and

$$
\operatorname{def}\left[\prod_{j=1}^{n} T_{0}\left(\tau_{j}\right)\right]=\sum_{j=1}^{n} \operatorname{def}\left[T_{0}\left(\tau_{j}\right)\right] ;
$$

(ii) the operators $T_{0}\left(\tau_{1} \tau_{2} \cdots \tau_{n}\right)$ and $\prod_{j=1}^{n}\left[T_{0}\left(\tau_{j}\right)\right]$ are not equal in general, that is,

$$
\left[T_{0}\left(\tau_{1} \tau_{2} \cdots \tau_{n}\right)\right] \subseteq \prod_{j=1}^{n}\left[T_{0}\left(\tau_{j}\right)\right]
$$

For symmetric differential operator $T_{0}\left(\tau_{j}\right)$, which satisfies property $(C)$, and by (2.23), (3.2) is constant on [0,2n]. In the problem with one singular endpoint, this constant is in [ $n, 2 n]$, while in the regular problem, it is equal to $2 n$, see [2]. 
LEMMA 3.2 (cf. [4, Theorem 2]). Let $\tau_{1}, \tau_{2}, \ldots, \tau_{n}$ be regular differential expressions on $[a, b]$. Suppose that $T_{0}\left(\tau_{j}\right)$ satisfies property $(C)$ for $j=1,2, \ldots, n$. Then,

$$
T_{0}\left(\tau_{1} \tau_{2} \cdots \tau_{n}\right)=\prod_{j=1}^{n} T_{0}\left(\tau_{j}\right)
$$

if and only if the following partial-separation condition is satisfied:

$$
\left\{f \in L_{w}^{2}(a, b), f^{[s-1]} \in \operatorname{AC}_{\mathrm{loc}}[a, b),\right.
$$

$$
\text { where } s \text { is the order of product expression }\left(\tau_{1} \tau_{2} \cdots \tau_{n}\right)
$$$$
\text { and }\left(\tau_{1} \tau_{2} \cdots \tau_{n}\right)^{+} f \in L_{w}^{2}(a, b) \text {, together imply that }
$$

$$
\left.\left(\prod_{j=1}^{k}\left(\tau_{j}^{+}\right)\right) f \in L_{w}^{2}(a, b), \quad k=1, \ldots, n-1\right\} \text {. }
$$

Therefore, (3.4) and (3.5) are equivalent.

We will say that the product $\left(\tau_{1} \tau_{2} \cdots \tau_{n}\right)$ is a partially separated expression in $L_{w}^{2}(a, b)$ whenever property (3.5) holds.

LEMMA 3.3. Let $\tau_{j}$ be a regular differential expression on $[a, b]$ for $j=$ $1, \ldots, n$. If all the solutions of the differential equation $\left(\tau_{j}\right) u=0$ and $\left(\tau_{j}^{+}\right) z=0$ on $[a, b]$ are in $L_{w}^{2}(a, b)$ for $j=1, \ldots, n$, then all the solutions of $\left(\tau_{1} \tau_{2} \cdots \tau_{n}\right) y=$ 0 and $\left(\tau_{1} \tau_{2} \cdots \tau_{n}\right)^{+} z=0$ are in $L_{w}^{2}(a, b)$.

Proof. Let $2=$ order of $\tau_{j}$, for $j=1, \ldots, n$. Then, $\operatorname{def}\left[T_{0}\left(\tau_{j}\right)\right]=2$. Hence, $T_{0}\left(\tau_{j}\right)$ has property $(C)$. By Lemma 3.1, we have

$$
\operatorname{def}\left[T_{0}\left(\tau_{1} \tau_{2} \cdots \tau_{n}\right)\right] \geq \operatorname{def}\left[\prod_{j=1}^{n} T_{0}\left(\tau_{j}\right)\right]=2 n=\text { order of }\left(\tau_{1} \tau_{2} \cdots \tau_{n}\right)
$$

Thus, def $\left[T_{0}\left(\tau_{1} \tau_{2} \cdots \tau_{n}\right)\right]=$ order of $\left(\tau_{1} \tau_{2} \cdots \tau_{n}\right)$, and, consequently, all the solutions of $\left(\prod_{j=1}^{n} \tau_{j}\right) y=0$ are in $L_{w}^{2}(a, b)$; we refer to [4] for more details.

The special case of Lemma 3.3 when $\tau_{j}=\tau$ for $j=1,2, \ldots, n$ and $\tau$ is symmetric was established in [16]. In this case, it is easy to see that the converse also holds. If all the solutions of $\tau^{n} y=0$ are in $L_{w}^{2}(a, b)$, then all the solutions of $\tau y=0$ must be in $L_{w}^{2}(a, b)$. In general, if all the solutions of $\left(\tau_{1} \tau_{2} \cdots \tau_{n}\right) y=0$ are in $L_{w}^{2}(a, b)$, then all the solutions of $\tau_{n} y=0$ are in $L_{w}^{2}(a, b)$ since these also are solutions of $\left(\tau_{1} \tau_{2} \cdots \tau_{n}\right) y=0$. If all the solutions of the adjoints equation $\left(\tau_{1} \tau_{2} \cdots \tau_{n}\right)^{+} z=0$ are also in $L_{n}^{2}(0, b)$, then it follows similarly that all the solutions of $\tau_{1}^{+} z=0$ are in $L_{n}^{2}(a, b)$. So, for $n=2$ in particular, we have established the following corollary. 
COROLLARY 3.4. Suppose that $\tau_{1}, \tau_{2}$, and $\tau_{1} \tau_{2}$ are all regular symmetric expressions on $[a, b)$. Then, the product is in the maximal deficiency case at $b$ if and only if both $\tau_{1}$ and $\tau_{2}$ are in the maximal deficiency case at $b$ (i.e., if $\tau_{1}$ and $\tau_{2}$ are in the classical limit-circle case at $b$, then the fourth-order expression $\tau_{1} \tau_{2}$ is in the limit-circle case at b; that is, $d^{+}=d^{-}=4$ ); see [4, Corollary 2] for more details.

In connection with the application of Lemma 3.1 to get information about the deficiency indices of symmetric differential expressions, we note that the product of symmetric expressions is not symmetric in general. However, any power of a symmetric expression is symmetric and so is called symmetric such as $\tau_{1} \tau_{2} \tau_{1}, \tau_{1} \tau_{2} T_{3} \tau_{2} \tau_{1}$, and so forth, of symmetric expressions are symmetric.

REMARK 3.5. In the case of product operators, the sesquilinear (bilinear) form $[f, g]$ can be written similar to that in (2.15) and (2.20) as follows: for $f, g \in D\left(\tau_{1} \tau_{2} \cdots \tau_{n}\right)$,

$$
\begin{aligned}
{[f, g] } & (x) \\
& =\sum_{k=1}^{n}(-1)^{(k-1)}\left(f^{[k-1]} \bar{g}^{[2 n-k]}-f^{[2 n-k]} \bar{g}^{[k-1]}\right)(x) \\
& =\left(\bar{g}, \bar{g}^{[1]}, \ldots, \bar{g}^{[2 n-1]}\right) J_{2 n \times 2 n}\left(f, f^{[1]}, \ldots, f^{[2 n-1]}\right)^{\mathrm{T}}(x) \\
& =\left(\left[\bar{g}, \phi_{1}\right],\left[\overline{\mathfrak{g}}, \phi_{2}\right], \ldots,\left[\bar{g}, \phi_{2 n}\right]\right) J_{2 n \times 2 n}\left(\left[f, \phi_{1}\right],\left[f, \phi_{2}\right], \ldots,\left[f, \phi_{2 n}\right]\right)^{\mathrm{T}}(x),
\end{aligned}
$$

T for transposed matrix, where $f^{[2 n-k]}, k=1, \ldots, 2 n$, are the quasiderivatives of $f, J_{2 n \times 2 n}=\left((-1)^{r} \delta_{r, 2 n+1-s}\right)(1 \leq r, s \leq 2 n)$ and $\phi_{1}, \phi_{2}, \ldots, \phi_{2 n}$ are linearly independent solutions of the equation $\left[\Pi_{j=1}^{n}\left(\tau_{j}\right)\right] u=0$. We refer to $[7,10,11]$ for more details.

The next result is a straightforward extension of [13, Section 18.1, Theorem $4]$, see also $[2,6,7]$.

THEOREM 3.6. If the operator $S$ with $D(S)$ is a selfadjoint extension of the minimal operator $T_{0}\left(\tau_{1} \tau_{2} \cdots \tau_{n}\right)=\prod_{j=1}^{n}\left[T_{0}\left(\tau_{j}\right)\right]$ with $\operatorname{def}\left[\prod_{j=1}^{n} T_{0}\left(\tau_{j}\right)\right]=d \in$ $[0,2 n]$, then there exist $\Psi_{1}, \ldots, \Psi_{d}$ in $D(S) \subset D\left[T\left(\tau_{1} \tau_{2} \cdots \tau_{n}\right)\right]$ satisfying the following conditions:

(i) $\Psi_{1}, \ldots, \Psi_{d}$ are linearly independent modulo $D\left[T_{0}\left(\tau_{1} \tau_{2} \cdots \tau_{n}\right)\right]$;

(ii) the sesquilinear form

$$
\left[\Psi_{j}, \Psi_{k}\right]_{a}^{b}=0, \quad j, k=1, \ldots, d
$$

(iii) $D(S)$ consists precisely of those $y$ in $D\left[T\left(\tau_{1} \tau_{2} \cdots \tau_{n}\right)\right]$ which satisfy

$$
\left[y, \Psi_{j}\right]_{a}^{b}=0, \quad j=1, \ldots, d .
$$


Conversely, given $\Psi_{1}, \ldots, \Psi_{d}$ in $D\left[T\left(\tau_{1} \tau_{2} \cdots \tau_{n}\right)\right]$ which satisfy (i) and (ii), the set $D(S)$ defined by (iii) is a selfadjoint domain.

PROoF. The proof is entirely similar to that in [13, Theorem 18.1.4] and therefore omitted.

REMARK 3.7. It is well known from Naimark [13] that no boundary condition is needed for a limit-point endpoint in order to get a selfadjoint realization of $\prod_{j=1}^{n}\left(\tau_{j}\right) u=0$. If both endpoints are LP, then no boundary conditions are necessary and hence the minimal (maximal) operator associated with $\prod_{j=1}^{n}\left(\tau_{j}\right)$ in $L_{w}^{2}(a, b)$ is itself selfadjoint and has no proper selfadjoint extensions (restrictions). On the other hand, a boundary condition is needed for each limit-circle endpoint.

The selfadjoint extensions are determined by boundary conditions imposed at the endpoints of the interval $I$. The type of these boundary conditions depends on the nature of the problem in the interval $I$.

THEOREM 3.8. Let $\tau_{1}, \tau_{2}, \ldots, \tau_{n}$ be a regular symmetric differential expressions on $[a, b]$, then the domain $D(S)$ of selfadjoint extension $S$ of $T_{0}\left(\tau_{1} \tau_{2} \cdots \tau_{n}\right)=\prod_{j=1}^{n}\left[T_{0}\left(\tau_{j}\right)\right]$ with $\operatorname{def}\left[\prod_{j=1}^{n} T_{0}\left(\tau_{j}\right)\right]=2 n$ is the set of functions $y \in D\left[T\left(\tau_{1} \tau_{2} \cdots \tau_{n}\right)\right]$ which are such that

$$
M Y(a)+N Y(b)=0
$$

where

$$
M=\left(\alpha_{j k}\right)_{1 \leq j, k \leq 2 n}, \quad N=\left(\beta_{j k}\right)_{1 \leq j, k \leq 2 n}
$$

are $2 n \times 2 n$ matrices over $\mathbb{C}, Y(\cdot)=\left(y, y^{[1]}, \ldots, y^{[2 n-1]}\right)^{\mathrm{T}}(\cdot)$, T for transposed matrix, and $\alpha_{j k}$ and $\beta_{j k}$ are complex numbers satisfying

$$
M J M^{*}=N J N^{*}, \quad J_{2 n \times 2 n}=(-1)^{r} \delta_{r, 2 n \cdots+1-s} \quad(1 \leq r, s \leq 2 n) .
$$

Conversely, if $S$ is a selfadjoint extension of $T_{0}\left(\tau_{1} \tau_{2} \cdots \tau_{n}\right)$, then there exist $2 n \times 2 n$ matrices $M$ and $N$ over $\mathbb{C}$ such that conditions (3.10) and (3.12) are satisfied and $D(S)$ is the set of functions $y \in D\left[T\left(\tau_{1} \tau_{2} \cdots \tau_{n}\right)\right]$ satisfying (3.10).

PROof. Let the boundary conditions (3.10) and (3.12) be given. By Theorem 2.5 , there are functions $\Psi_{1}, \ldots, \Psi_{2 n}$ in $D\left[T\left(\tau_{1} \tau_{2} \cdots \tau_{n}\right)\right]$ which satisfy the conditions

$$
\bar{\Psi}_{j}^{[2 n-k]}(a)=(-1)^{k} \alpha_{j k}, \quad \bar{\Psi}_{j}^{[2 n-k]}(b)=(-1)^{(k-1)} \beta_{j k}, \quad j, k=1, \ldots, 2 n .
$$

Given (3.13), it is not difficult to show that (3.12) and (3.10) can be restated in forms (3.8) and (3.9), respectively. It then follows from Theorem 3.6 that the domain determined by (3.10) and (3.12) is the domain of selfadjoint extension of $T_{0}\left(\tau_{1} \tau_{2} \cdots \tau_{n}\right)$. 
Conversely, if $S$ is a selfadjoint extension of $T_{0}\left(\tau_{1} \tau_{2} \cdots \tau_{n}\right)$, then, by Theorem 3.6, $D(S)$ is determined by the functions $\Psi_{1}, \ldots, \Psi_{2 n}$ in $D\left[T\left(\tau_{1} \tau_{2} \cdots \tau_{n}\right)\right]$ satisfying (3.8) and (3.9). If $\alpha_{j k}$ and $\beta_{j k}, 1 \leq j, k \leq 2 n$ are then defined by (3.13), it is clear that $D(S)$ is determined by $(3.10)$ and $(3.12)$, see $[7,8,13]$ for more details.

In the following cases, the selfadjoint extension $S$ of $T_{0}\left(\tau_{1} \tau_{2} \cdots \tau_{n}\right)$ is determined by boundary conditions in terms of certain Wronskians (sesquilinear forms) involving $y$ and $2 n$ linearly independent solutions of the equation $\left(\prod_{j=1}^{n} \tau_{j}\right) u=0$ at the singular endpoints.

CASE (i). Assume that both endpoints $a$ and $b$ are singular LC. By (3.7), (3.8), and Lemma 2.6, if we put

$$
\left[\bar{\Psi}_{j}, \phi_{k}\right](a)=(-1)^{k} \alpha_{j k}, \quad\left[\bar{\Psi}_{j}, \phi_{k}\right](a)(b)=(-1)^{(k-1)} \beta_{j k}, \quad j, k=1, \ldots, 2 n,
$$

then the boundary conditions of the function $y \in D\left[T\left(\tau_{1} \tau_{2} \cdots \tau_{n}\right)\right]$ have the same form (3.10), where $M, N$ satisfy (3.11) and (3.12), and $Y(\cdot)=\left(\left[y, \phi_{1}\right]\right.$, $\left.\ldots,\left[y, \phi_{2 n}\right]\right)^{\mathrm{T}}(\cdot)$.

CASE (ii). (a) Assume that the left endpoint $a$ is regular and the right endpoint $b$ is singular LC. Then, the boundary conditions of the functions $y \in$ $D\left[T\left(\tau_{1} \tau_{2} \cdots \tau_{n}\right)\right]$ in this case are given by (3.10), where

$$
\begin{aligned}
& Y(a)=\left(y, y^{[1]}, \ldots, y^{[2 n-1]}\right)^{\mathrm{T}}(a), \\
& Y(b)=\left(\left[y, \phi_{1}\right], \ldots,\left[y, \phi_{2 n}\right]\right)^{\mathrm{T}}(b),
\end{aligned}
$$

and the matrices $M$ and $N$ satisfy (3.12).

(b) If the left endpoint $a$ is singular LC and the right endpoint $b$ is regular, then let

$$
\begin{aligned}
& Y(a)=\left(\left[y, \phi_{1}\right], \ldots,\left[y, \phi_{2 n}\right]\right)^{\mathrm{T}}(a), \\
& Y(b)=\left(y, y^{[1]}, \ldots, y^{[2 n-1]}\right)^{\mathrm{T}}(b),
\end{aligned}
$$

and the rest is the same as in (a).

CASE (iii). Assume that one endpoint is LP endpoint and the other is either regular or singular LC endpoint, then we have

(a) suppose $a$ is LP. Then, the boundary conditions in this case on the functions $y \in D\left[T\left(\tau_{1} \tau_{2} \cdots \tau_{n}\right)\right]$ are (3.10) with $M=0$; that is,

$$
N Y(b)=0,
$$

where

$Y(b)=\left(y, y^{[1]}, \ldots, y^{[2 n-1]}\right)^{\mathrm{T}}(b), \quad$ if $b$ is regular, $Y(b)=\left(\left[y, \phi_{1}\right], \ldots,\left[y, \phi_{2 n}\right]\right)^{\mathrm{T}}(b), \quad$ if $b$ is singular and LC; 
(b) if $b$ is LP, then it suffices to reverse the roles of $a$ and $b$ in (a).

CASE (iv). If both endpoints $a$ and $b$ are LP, then no boundary conditions are necessary, see Remark 3.7.

4. Discussion. In this section, we show how Cases (i), (ii), (iii), and (iv) follow from the sesquilinear form (3.7), Lemma 2.6, and Theorem 3.6. The cases $d=0$, $n, 2 n$ are considered separately.

CASE $1(d=0)$. In this case, both endpoints are LP endpoints and the minimal operator $T_{0}\left(\tau_{1} \tau_{2} \cdots \tau_{n}\right)$ is itself selfadjoint and has no proper selfadjoint extensions.

CASE $2(d=n)$. In this case, one endpoint must be LP and the other either regular or LC endpoint.

(2a) Assume that $a$ is LP and $b$ is regular. In this case, condition (iii) of Theorem 3.6 becomes

$$
\begin{aligned}
{\left[y, \Psi_{j}\right]_{a}^{b} } & =\left[y, \Psi_{j}\right](b) \\
& =\sum_{k=1}^{n}(-1)^{(k-1)}\left[y^{[k-1]} \bar{\Psi}_{j}^{[2 n-k]}-y^{[2 n-k]} \bar{\Psi}_{j}^{[k-1]}\right](b) \\
& =0, \quad j=1, \ldots, n .
\end{aligned}
$$

If $b$ is regular, then $\Psi_{j}(b), \Psi_{j}^{[1]}(b), \ldots, \Psi_{j}^{[2 n-1]}(b)$ can take an arbitrary values and so (3.10) can be rewritten as

$$
N Y(b)=0,
$$

where $N=\left(\beta_{j k}\right)_{1 \leq j \leq n, 1 \leq k \leq 2 n}$ and $Y(b)=\left(y, y^{[1]}, \ldots, y^{[2 n-1]}\right)^{\mathrm{T}}(b)$.

From Theorem 3.6(i), it follows that not all of $\beta_{j, 1}, \ldots, \beta_{j, 2 n}$ can be zero since this would imply, by Theorem 3.6, that $\Psi_{j} \in D_{0}\left(\tau_{1} \tau_{2} \cdots \tau_{n}\right)$ and $j=1, \ldots, n$. condition (ii) becomes

$$
N J_{2 n \times 2 n} N^{*}=0, \quad J_{2 n \times 2 n}=(-1)^{r} \delta_{r, 2 n \cdots+1-s} \quad(1 \leq r, s \leq 2 n) .
$$

Hence, the selfadjoint boundary conditions are of the form (4.2) with real $\beta_{j, 1}, \ldots, \beta_{j, 2 n}$, not all zero $j=1, \ldots, n$.

We have similar result if $a$ is regular and $b$ is LP.

(2b) Assume that $a$ is LP and $b$ is LC. In this case, condition (iii) becomes (4.1), which is equivalent to

$$
\left(\left[\bar{\Psi}_{j}, \phi_{1}\right], \ldots,\left[\bar{\Psi}_{j}, \phi_{2 n}\right]\right) J_{2 n \times 2 n}\left(\left[y, \phi_{1}\right], \ldots,\left[y, \phi_{2 n}\right]\right)^{\mathrm{T}}=0, \quad j=1, \ldots, n .
$$

Set

$$
\left[\bar{\Psi}_{j}, \phi_{k}\right](b)=(-1)^{(k-1)} \beta_{j k}, \quad j=1, \ldots, n ; k=1, \ldots, 2 n .
$$


Then, the selfadjoint boundary conditions (iii) can be expressed as

$$
N Y(b)=0
$$

where $N=\left(\beta_{j k}\right)_{1 \leq j \leq n, 1 \leq k \leq 2 n}$ and $Y(b)=\left(\left[y, \phi_{1}\right], \ldots,\left[y, \phi_{2 n}\right]\right)^{\mathrm{T}}(b)$. Again, by Theorem 3.6(i), $\beta_{j, 1}, \ldots, \beta_{j, 2 n}, j=1, \ldots, n$ are real and not all zero.

Similarly, for the case when $a$ is LC and $b$ is LP.

REMARK 4.1. Assume that $a$ is LP. Comparing (4.6) with (4.2), note that when $y^{[k-1]}(b)$ is replaced by $\left[y, \phi_{k}\right](b), k=1, \ldots, 2 n$, then the singular case when $b$ is LC is an exact parallel to the case when $b$ is regular.

CASE $3(d=2 n)$. In this case, each endpoint is either regular or LC. By (3.10), (3.13) and proceeding as in Case 2, we find that condition (iii) is equivalent to the equations

$$
\sum_{k=1}^{2 n} \alpha_{j k}\left[y, \phi_{k}\right](a)+\sum_{k=1}^{2 n} \beta_{j k}\left[y, \phi_{k}\right](b)=0, \quad j=1, \ldots, 2 n .
$$

Theorem 3.6(i) guarantees the linear independence of $2 n$ equations in (4.7), and condition (ii) reduces to the following conditions:

$$
\begin{aligned}
& \sum_{s=1}^{n} \alpha_{j s} \bar{\alpha}_{k, 2 n-s+1}-\sum_{s=1}^{n} \alpha_{j, 2 n-s+1} \bar{\alpha}_{k s} \\
& \quad=\sum_{s=1}^{n} \beta_{j s} \bar{\beta}_{k, 2 n-s+1}-\sum_{s=1}^{n} \beta_{j, 2 n-s+1} \bar{\beta}_{k s}, \quad j, k=1, \ldots, 2 n .
\end{aligned}
$$

We refer to $[5,6,7,10]$ for more details.

REMARK 4.2. It remains an open question as to characterize the singular non selfadjoint boundary conditions provided that $y$ and its quasiderivatives are replaced by certain Wronskians (sesquilinear form) associated with nonsymmetric differential expressions involving $y$ and elements of the maximal domain.

\section{REFERENCES}

[1] J. Chaudhuri and W. N. Everitt, On the square of a formally self-adjoint differential expression, J. London Math. Soc. (2) 1 (1969), 661-673.

[2] D. E. Edmunds and W. D. Evans, Spectral Theory and Differential Operators, Oxford Mathematical Monographs, Oxford University Press, New York, 1987.

[3] W. N. Everitt and M. Giertz, On some properties of the powers of a formally selfadjoint differential expression, Proc. London Math. Soc. (3) 24 (1972), 149170.

[4] W. N. Everitt and A. Zettl, The number of integrable-square solutions of products of differential expression, Proc. Roy. Soc. Edinburgh Sect. A 76 (1977), 215226. 

, Sturm-Liouville differential operators in direct sum spaces, Rocky Mountain J. Math. 16 (1986), no. 3, 497-516.

[6] D. Hinton, A. M. Krall, and K. Shaw, Boundary conditions for differential operators with intermediate deficiency index, Appl. Anal. 25 (1987), no. 1-2, 43-53.

[7] S. E. Ibrahim, On boundary conditions for Sturm-Liouville differential operators in the direct sum spaces, Rocky Mountain J. Math. 29 (1999), no. 3, 873-892.

[8] _ , The products of general quasi-differential operators and their essential spectra, Int. J. Appl. Math. 1 (1999), no. 7, 725-756.

[9] _ - The point spectra and regularity fields of products of quasi-differential operators, Indian J. Pure Appl. Math. 31 (2000), no. 6, 647-665.

[10] A. M. Krall and A. Zettl, Singular selfadjoint Sturm-Liouville problems, Differential Integral Equations 1 (1988), no. 4, 423-432.

[11]_ Singular selfadjoint Sturm-Liouville problems. II. Interior singular points, SIAM J. Math. Anal. 19 (1988), no. 5, 1135-1141.

[12] S. J. Lee, On boundary conditions for ordinary linear differential operators, J. London Math. Soc. (2) 12 (1975/1976), no. 4, 447-454.

[13] M. A. Naimark, Linear Differential Operators. Part II: Linear Differential Operators in Hilbert Space, Frederick Ungar Publishing, New York, 1968.

[14] P. W. Walker, A note on differential equations with all solutions of integrablesquare, Pacific J. Math. 56 (1975), no. 1, 285-289.

[15] A. Zettl, Deficiency indices of polynomials in symmetric differential expressions, Ordinary and Partial Differential Equations (Proc. Conf., Univ. Dundee, Dundee, 1974), Lecture Notes in Mathematics, vol. 415, Springer, Berlin, 1974, pp. 293-301.

[16]___ Deficiency indices of polynomials in symmetric differential expressions. II, Proc. Roy. Soc. Edinburgh Sect. A 73 (1975), 301-306.

[17] _ Formally self-adjoint quasi-differential operators, Rocky Mountain J. Math. 5 (1975), no. 3, 453-474.

[18] _ The limit-point and limit-circle cases for polynomials in a differential operator, Proc. Roy. Soc. Edinburgh Sect. A 72 (1975), no. 3, 219-224.

Sobhy El-Sayed Ibrahim: Department of Mathematics, Faculty of Science, Benha University, Benha 13518, Kalubia, Egypt 


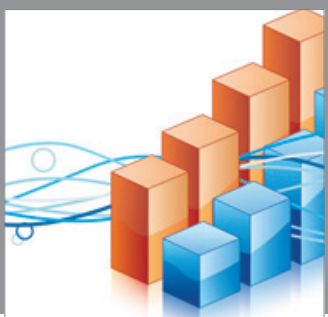

Advances in

Operations Research

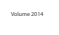

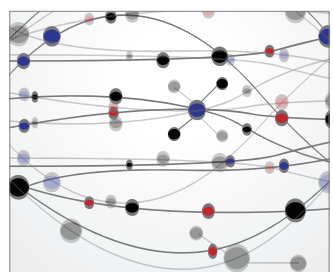

\section{The Scientific} World Journal
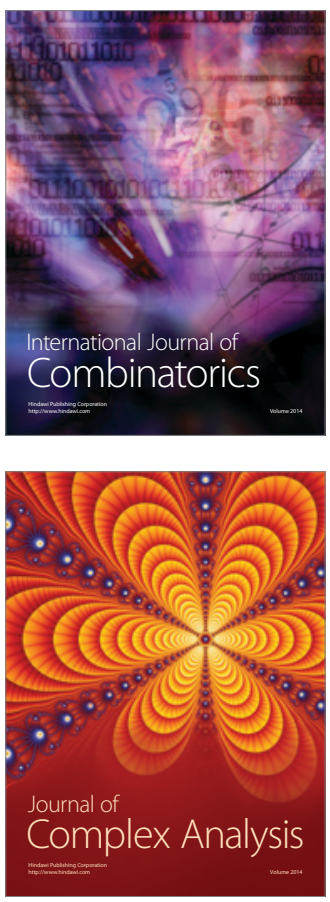

International Journal of

Mathematics and

Mathematical

Sciences
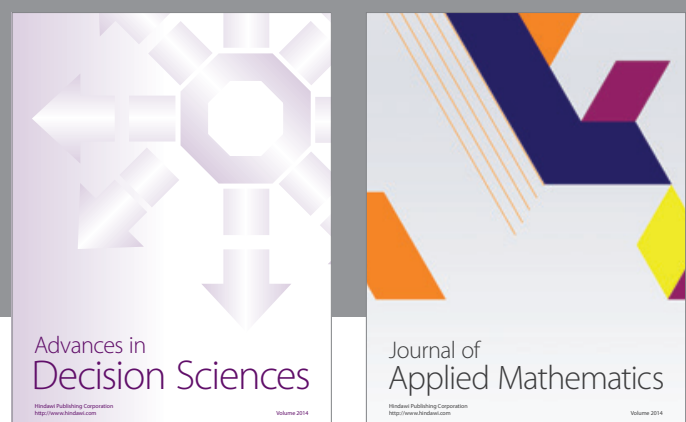

Journal of

Applied Mathematics
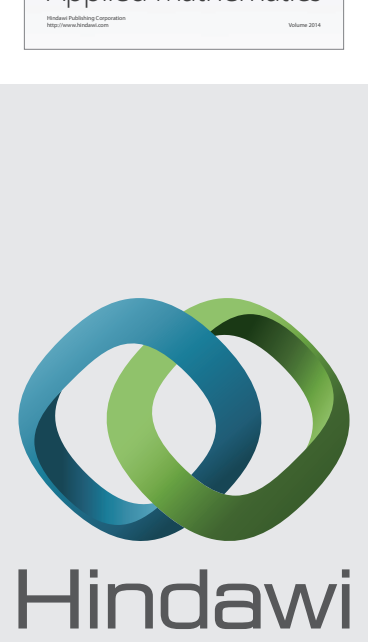

Submit your manuscripts at http://www.hindawi.com
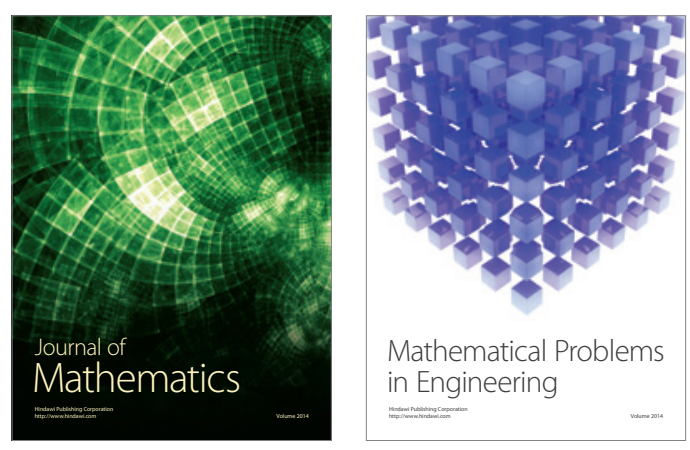

Mathematical Problems in Engineering
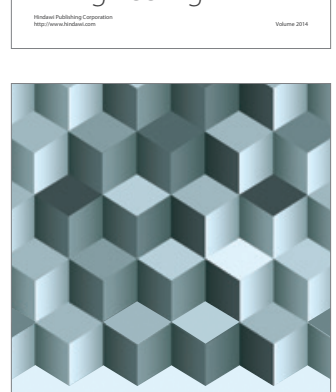

Journal of

Function Spaces
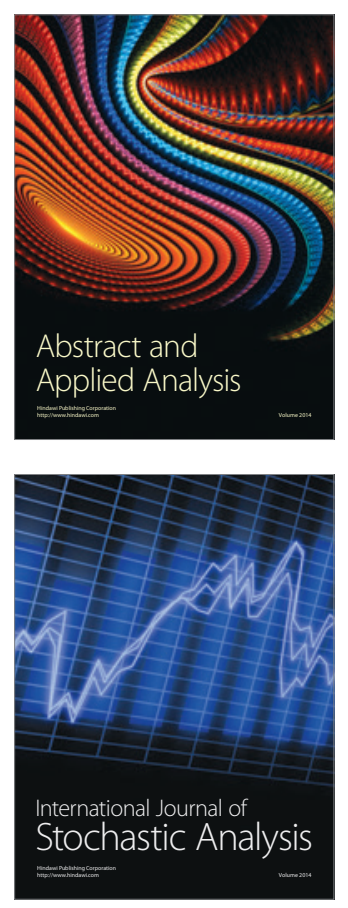

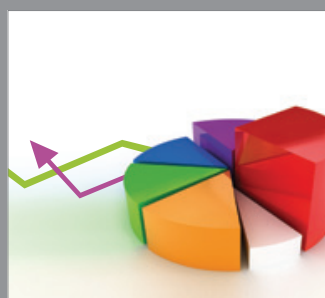

ournal of

Probability and Statistics

Promensencen
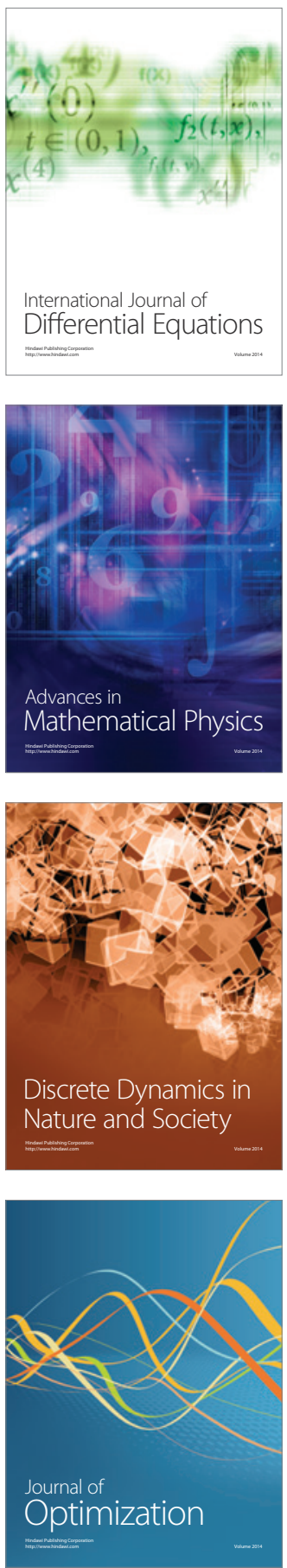